\title{
Recent Developments on Mobile Ad-Hoc Networks and Vehicular Ad-Hoc Networks
}

\author{
Dimitris Kanellopoulos $1, *$ (D) and Francesca Cuomo $2, *$ (D) \\ 1 Department of Mathematics, University of Patras, GR 26500 Patras, Greece \\ 2 Department of Information Engineering, Electronics and Telecommunications, Sapienza University of Rome, \\ 00184 Rome, Italy \\ * Correspondence: d_kan2006@yahoo.gr (D.K.); francesca.cuomo@uniroma1.it (F.C.)
}

Citation: Kanellopoulos, D.; Cuomo, F. Recent Developments on Mobile Ad-Hoc Networks and Vehicular Ad-Hoc Networks. Electronics 2021, 10, 364. https://doi.org/10.3390/ electronics10040364

Academic Editor: Juan-Carlos Cano Received: 28 January 2021

Accepted: 1 February 2021

Published: 3 February 202

Publisher's Note: MDPI stays neutral with regard to jurisdictional claims in published maps and institutional affiliations.

Copyright: (c) 2021 by the authors. Licensee MDPI, Basel, Switzerland. This article is an open access article distributed under the terms and conditions of the Creative Commons Attribution (CC BY) license (https:/ / creativecommons.org/licenses/by/ $4.0 /)$.

\section{Introduction}

Mobile ad-hoc networks (MANETs) have a decentralized nature that makes them suitable for a variety of applications. The main advantage of a MANET [1] is that its nodes can communicate without any infrastructure. As a result, MANETs are usually deployed in battlefields, natural disasters, etc. MANETs differ from the long-established computer networks, as they have unique characteristics. For example, in a MANET, we observe a frequent link breakage because of node mobility, a high channel-error rate, severe link-layer contentions, and different path properties such as delay, bandwidth, and packet loss rate. Due to these characteristics, the overall performance of a MANET is disturbed in terms of packet delivery ratio, end-to-end delay, network throughput, and network overhead. By applying the principles of MANETs, a vehicular ad-hoc network (VANET) [2] can be established in an ad-hoc mode by vehicles. Using a VANET, vehicles can directly communicate among them, with no supporting infrastructure. Besides, VANETs are employed for road monitoring and infotainment applications, which constitute an integral part of the intelligent transportation system paradigm.

This Special Issue, entitled "Recent Developments on Mobile Ad-Hoc Networks and Vehicular Ad-Hoc Networks", aims to expose the readership to the latest novel works in terms of solutions and techniques for MANETs and VANETs. The Special Issue is composed of five referred papers, covering interesting topics such as power-aware optimization solutions for MANETs, data dissemination in VANETs, adaptive multi-hop broadcast schemes for VANETs, multi-metric routing protocols for VANETs, and incentive mechanisms to encourage the distribution of information in VANETs. The Issue sets out to demonstrate pioneering work in these fields, investigate novel solutions and methods, and discuss future trends in these fields.

\section{The Papers in this Special Issue}

Using the expected utility theory of conventional economics, we can introduce rewardbased incentive mechanisms to enable vehicle nodes to share information in a VANET. These mechanisms often assume that the positive and negative effects (formed by an equivalent quantity of gain and loss) are equal in terms of absolute value. Nevertheless, the theory of loss aversion indicates that the above effects are not equal. Moreover, this can result in a divergence between the last decision-making behaviors of vehicle nodes and the real, most favorable situation.

In the first paper [3], the authors propose a Loss-Aversion-based Incentive Mechanism (LAIM) to encourage the complete awareness and sharing of information in VANETs. To stimulate the cooperation among vehicle nodes, the authors designed the incentive threshold and the threshold factor. The authors redesigned the utility function of nodes to correct the assumption that a gain and a loss of an equal quantity could equalize each other in conventional economics. The simulation results demonstrate that the LAIM mechanism 
can increase the average utility of nodes by more than $34.35 \%$. This result, in turn, promotes the cooperation of vehicle nodes.

In the past decade, data dissemination in VANETs has attracted much attention, as it is a crucial function for the well-organized exchange of traffic and road information. In VANETs, a research challenge is to propose a multi-hop broadcast scheme that will be adaptive and obtain high reachability whilst utilizing the bandwidth by avoiding redundant transmissions. In the light of this context, the authors of the second paper [4] introduce an intelligent fuzzy logic-based density and distribution adaptive broadcast protocol for VANETs. The new protocol calculates the spatial distribution of vehicles in the network. To enhance reachability, the new protocol uses the Nearest Neighbor Distance method to adapt the transmission range. This adapts the contention window size to the network density and spatial distribution, and thus decreases packet collisions. It also uses the Bloom filter technique to decrease the overhead resulting from the inclusion of the neighbor IDs in the header of the broadcast message. The simulation results demonstrate that the new protocol is effective, as it improves reachability and the ability to utilize bandwidth.

In the third paper [5], the authors introduce an epidemic algorithm (EPIC) for message dissemination in VANETs. The main design goal of the EPIC is "to have the highest number of vehicles "infected" by the message, without overloading the network" [5]. From the literature on the connected cover set, the authors derive a theoretical bound. Then, they compare EPIC with this bound to evaluate the near-optimality of EPIC. It is well-known that ETSI ITS-G5 GeoNetworking is one standard of vehicular communication. In this standard, one of the basic forwarding methods is Contention-Based Forwarding (CBF). As a consequence, the authors compare EPIC with $\mathrm{CBF}$ and $\mathrm{CBF}+$ (an evolution of $\mathrm{CBF}$ ) and prove that, in real urban scenarios, the performance gain of the EPIC algorithm attains a satisfactory result. For example, EPIC can reach many vehicles and involve a small number of relay vehicle nodes.

Moreover, the research community has proposed a plethora of power-aware optimization solutions for MANETs. Such solutions are energy-efficient proactive (link state-based) and reactive (source-initiated-based) routing protocols, routing protocols based on adaptive load balancing, location-based routing protocols, multicast-based and cross-layer based routing protocols, transmission power control-based routing protocols, and approaches based on adaptations of the radio state operational mode. The fourth paper [6] considers key issues on power-aware optimization solutions for MANETs and classifies the existing power-efficient optimization solutions for MANETs into eight categories. The authors extensively review these solutions and discuss open research directions in the field, such as hybrid optimization algorithms for topology management in cluster-based MANETs and the design of cooperative MAC protocols.

From another perspective, existing routing protocols for VANETs often use several metrics to select the best route in a VANET. Such metrics are speed, position, distance, density, and link stability. In the fifth paper [7], the authors present an analysis of those routing protocols which are based on multiple metrics. The authors describe the most often-used metrics in various routing schemes for VANETs and their application scenarios. Their survey employs a systematic literature review methodology that allows them to study the existing high-tech routing schemes for VANETs. In their survey, the authors also confirm that speed and distance are the most accepted and flexible metrics.

Many research challenges have yet to be addressed by the community. For example, it is vital to invent and test new mathematical models [8] for the flooding techniques applied to famous power-efficient routing protocols for MANETs and VANETs. This direction may assist the research community in introducing novel energy-aware flooding methods in ad-hoc networks.

To conclude, we hope the reader will find this Special Issue useful for future research activities in MANETs and VANETs, as well in emerging paradigms related to these topics.

Funding: This research received no external funding. 
Acknowledgments: We would like to thank all researchers who submitted articles to this special issue. We are also grateful to all reviewers who helped in the evaluation of the manuscripts and made very valuable suggestions to improve the quality of contributions. We would like to acknowledge the editorial board of Electronics, who invited us to guest edit this special issue. We are also grateful to the Electronics Editorial Office staff who worked thoroughly to maintain the rigorous peer-review schedule and timely publication.

Conflicts of Interest: The authors declare no conflict of interest.

\section{References}

1. Sarkar, S.K.; Basavaraju, T.G.; Puttamadappa, C. Ad Hoc Mobile Wireless Networks: Principles, Protocols, and Applications, 2nd ed.; CRC Press: Boca Raton, FL, USA, 2013; ISBN 978-1-4665-1446-1.

2. Karagiannis, G.; Altintas, O.; Ekici, E.; Heijenk, G.; Jarupan, B.; Lin, K.; Weil, T. Vehicular Networking: A Survey and Tutorial on Requirements, Architectures, Challenges, Standards and Solutions. IEEE Commun. Surv. Tutor. 2011, 13, 584-616. [CrossRef]

3. Liu, J.; Huang, S.; Xu, H.; Li, D.; Zhong, N.; Liu, H. Cooperation Promotion from the Perspective of Behavioral Economics: An Incentive Mechanism Based on Loss Aversion in Vehicular Ad-Hoc Networks. Electronics 2021, 10, 225. [CrossRef]

4. Limouchi, E.; Mahgoub, I. Smart Fuzzy Logic-Based Density and Distribution Adaptive Scheme for Efficient Data Dissemination in Vehicular Ad Hoc Networks. Electronics 2020, 9, 1297. [CrossRef]

5. Spadaccino, P.; Cuomo, F.; Baiocchi, A. Epidemic and Timer-Based Message Dissemination in VANETs: A Performance Comparison. Electronics 2020, 9, 595. [CrossRef]

6. Kanellopoulos, D.; Sharma, V.K. Survey on Power-Aware Optimization Solutions for MANETs. Electronics 2020, 9, 1129. [CrossRef]

7. Tripp-Barba, C.; Zaldívar-Colado, A.; Urquiza-Aguiar, L.; Aguilar-Calderón, J.A. Survey on Routing Protocols for Vehicular Ad Hoc Networks Based on Multimetrics. Electronics 2019, 8, 1177. [CrossRef]

8. Alzahrani, F.A. On Modeling Optimizations and Enhancing Routing Protocols for Wireless Multihop Networks. IEEE Access 2020, 8, 68953-68973. [CrossRef] 